\title{
Vena cava inferior como factor predictor del shock en trauma: medición ecográfica
}

\author{
Fernando Rey S. ${ }^{1}$, Erika Vásquez U. ${ }^{1}$ y José Félix Vivas A. ${ }^{1}$
}

\section{Inferior vena cava as a predictor of shock in trauma patients: sonographic measurement}

\begin{abstract}
Aim: To establish the sonographic measurement of the diameter of the inferior vena cava as a predictor of shock in trauma patients. Materials and Method: A cross-sectional study to determined the sonographic measurement of the inferior vena cava to 40 patients attended at Hospital Domingo Luciani trauma unit, in the period between January and April of 2018. Two groups were selected; group 1: shock patients, those with systolic blood pressure lower than $90 \mathrm{~mm} \mathrm{Hg}$, and the group 2: control patients that kept normal blood pressure. The image study of the inferior vena cava was carried out with the ALOKA prosound ultrasound equipment SSD a5 and with the portable ultrasound MicroMaxx SonoSite, in which the collapsibility index was measured. The findings were recorded in the data collection sheet. The statistical analysis proposed for the comparison of averages was made with the student's t-test for independent samples and the assessment of diagnostic cut-off points was made with a ROC curve. Results: the mean of collapsability index of de IVC for control and shock group were $26 \pm 12,7 \%$ and 58,5 $\pm 5,9 \%$, respectively; The collapsability index (CI) was $>50 \%$ in all patients of shock group. Conclusions: The measurement of the ICV diameter is a predictor of shock, being the IC the most sensitive and specific parameter.
\end{abstract}

Key words: shock; trauma; sonographic; inferior vena cava.

\section{Resumen}

Objetivo: Establecer la medición ecográfica del diámetro de la vena cava inferior como factor predictor del shock en pacientes politraumatizados. Materiales y Método: Estudio de corte transversal donde se determinó la medición ecográfica de la vena cava inferior a 40 pacientes que ingresaron a la Unidad de Politraumatizados (UPT) del Hospital General del Este "Dr. Domingo Luciani”, en el período entre enero y abril de 2018. Se seleccionaron 2 grupos; el grupo 1: pacientes en shock, aquellos con tensión arterial sistólica menor a $90 \mathrm{~mm} \mathrm{Hg}$ al ingreso, y el grupo 2: pacientes controles que mantuvieron cifras tensionales normales. El estudio de imagen de la vena cava inferior se realizó con el equipo de ecografía $A L O K A$ prosound SSD-a5 y con el ultrasonido portátil MicroMaxx SonoSite, en el cual se midió el índice de colapsabilidad. Los hallazgos se registraron en la hoja de recolección de datos. El análisis estadístico se hizo con la prueba de $t$ de student para muestras independientes y la valoración de puntos de corte diagnóstico se hizo con la prueba de ROC. Resultados: La media del Índice de colapsabilidad (IC) de la VCI para el grupo control y de shock fue de $26 \pm 12,7 \%$ y de 58,5 $\pm 5,9 \%$, respectivamente; El índice de colapsabilidad fue $>50 \%$ en todos los pacientes del grupo de shock. Conclusiones: La medición del diámetro de la VCI es un predictor de shock, siendo el IC el parámetro más sensible y específico.

Palabras clave: shock; trauma; ecografía; vena cava inferior.

\section{Introducción}

El manejo inicial del paciente politraumatizado es una situación clínica compleja donde se debe valorar y priorizar al paciente según el tipo de lesiones, la estabilidad de sus signos vitales y el mecanismo del trauma, por lo que se debe de seguir un esquema sistemático de evaluación, conocido como el ABCDEF del paciente politraumatizado: A) vía aérea y control de la columna cervical; B) ventilación; C) circulación y control de la hemorragia; D) déficit neurológico; E) exposición y control de la hipotermia; F) manejo del dolor ${ }^{1}$.
'Hospital General del Este Dr. Domingo Luciani, Caracas, Venezuela.

Recibido el 26 de enero de 2019 y aceptado para publicación el 26 de marzo de 2019.

Correspondencia a Dra. Erika Vásquez U. caridad.vas@gmail.com 
En la evaluación primaria en cirugía de trauma, específicamente en la valoración de la circulación y control de la hemorragia, el uso del ultrasonido se ha vuelto una herramienta indispensable, que además de ser de uso fácil y económico, tiene como objetivo principal identificar la presencia de líquido libre en la cavidad abdominal y pericárdico ${ }^{1}$.

Existen parámetros clínicos y paraclínicos para estimar el estado hemodinámico del paciente, tales como: nivel de conciencia, presión arterial media, llenado capilar, pulso y color y temperatura de la piel, permitiendo identificar la ausencia o presencia del shock en el paciente politraumatizado ${ }^{1}$.

Entre los parámetros de evaluación mencionados no existe ninguno que sea un indicador pronóstico fiable de la situación hemodinámica del paciente, que permita priorizar cada caso de forma objetiva, a fin de perfeccionar el manejo del paciente en la unidad de politraumatizados ${ }^{1}$.

La detección de la capacidad de respuesta al fluido es de suma importancia en el manejo de pacientes con shock hipovolémico, ya que permite mejorar el gasto cardíaco y por tanto la hemodinamia al administrar infusiones intravenosas de líquidos. Sin embargo, la reanimación volumétrica agresiva puede ser perjudicial en algunos pacientes con otro tipo de shock ${ }^{1}$.

Existen evidencias que demuestran que la administración excesiva de líquidos puede dar lugar a un peor resultado, causando edema pulmonar y reducción de la difusión de oxígeno. Desafortunadamente, la evaluación clínica por sí sola no es capaz de distinguir qué pacientes se beneficiarán de la administración de líquidos por vía intravenosa y qué pacientes pueden empeorar ${ }^{2}$.

El diagnóstico preciso del estado de shock puede ser un reto para el personal médico dificultando el tratamiento adecuado del mismo. Los catéteres en la arteria pulmonar y de la presión venosa central si bien proporcionan datos fisiológicos, tales como el gasto cardíaco y la presión auricular derecha consumen mucho tiempo, son invasivos y conllevan riesgos considerables como neumotórax, hemotórax (especialmente si se introducen por vía subclavia) embolias, trombosis venosas, arritmias, sepsis y punciones arteriales ${ }^{3}$.

Dada la importancia de determinar el volumen intravascular en pacientes en shock, la ecografía es una técnica sencilla y no invasiva, que ha demostrado ser un método adecuado para detectar las fuentes de sangrado en los pacientes con trauma. Un mejor indicador del volumen intravascular es el colapso de la vena cava inferior ( $\mathrm{VCI}$ ), que se contrae y se distiende con la presión y los cambios de volumen ${ }^{4}$.
Es por ello que se pretende determinar si con la medición ecográfica del diámetro de la vena cava inferior es posible estimar el riesgo de shock por hemorragia al ingreso a la unidad, permitiendo de esta manera una valoración del estado de shock sin necesidad de procedimientos invasivos.

La importancia del presente estudio radica en proporcionar una nueva herramienta fiable, no invasiva, fácil de realizar y de bajo costo para la evaluación del estado hemodinámico en la valoración primaria del politraumatizado, con el fin de optimizar la reanimación y administración eficaz de líquidos intravasculares, tratar precozmente la hemorragia y tener un criterio más objetivo al momento de la toma de decisiones según prioridades en un grupo de pacientes.

\section{Objetivo general}

Determinar la utilidad de la medición ecográfica de la VCI como factor predictor del shock hipovolémico en pacientes politraumatizados.

\section{Objetivos específicos}

1. Establecer las mediciones del diámetro de la VCI en inspiración y espiración en pacientes politraumatizados.

2. Evaluar la sensibilidad y especificidad de la medición del diámetro de la vena cava inferior en pacientes ingresados en la unidad de politraumatizados en el período comprendido entre enero y abril de 2018 .

3. Determinar el punto de corte óptimo del diámetro de la VCI que permite la correlación y predicción del estado de shock del paciente.

\section{Materiales y Método}

\section{Tipo de estudio}

La presente se trata de un diseño no experimental tipo transversal.

\section{Población y muestra}

La población incluyo a pacientes que ingresaron a la Unidad de Politraumatizado del Hospital General del Este "Dr. Domingo Luciani" ubicado en El Llanito, Caracas- Venezuela, en el periodo comprendido entre enero y abril del 2018. Se tomaron como muestra 20 pacientes control (PAS $\geq$ a $90 \mathrm{mmHg}$ ) y 20 en shock (PAS < a $90 \mathrm{mmHg}$ ) de 16 años o más, dentro de las primeras $6 \mathrm{~h}$ después de la admisión, que firmaron el consentimiento informado si estaban conscientes o de lo contrario firmó el familiar. Los criterios de exclusión toma- 
dos en cuenta fueron: 1) Pacientes que desarrollaron paro cardiopulmonar; 2) Pacientes que no fueron adecuados para la visualización por ultrasonido de VCI debido a problemas técnicos (obesidad severa, enfisema subcutáneo, herida en el epigastrio $\mathrm{u}$ otros problemas que dificultan la visualización óptima de VCI); 3) Embarazadas; 4) Paciente con problemas cardíacos congénitos y 5) Pacientes que ingresaron a la UPT después de transcurridas $12 \mathrm{~h}$ del traumatismo.

\section{Procedimientos}

La técnica que se usó para la recolección de datos fue bajo la modalidad de observación en donde los pacientes fueron incluidos y evaluados durante las primeras $6 \mathrm{~h}$ después de la admisión, previamente se habló con los familiares explicando el motivo del estudio y se entregó un consentimiento informado el cual debió ser firmado. Al ingreso se determinó la PAS y se registró. Posteriormente, se procedió a evaluar la distensibilidad de la VCI por ultrasonografía usando un equipo portátil MicroMaxx SonoSite o el equipo de ultrasonido del servicio de Radiodiagnóstico, marca ALOKA, modelo ProSound $\alpha 5$ Sv. La exploración mediante el US del diámetro de la vena cava inferior fue de la siguiente manera:

1. Se colocó al paciente en decúbito dorsal.

2. Se colocó el transductor convexo DE 2-5 MhZ en epigastrio, se localizó la ventana acústica subxifoidea o subcostal, colocando al transductor inmediatamente por debajo y ligeramente hacia la derecha del apéndice xifoides (Figura 1).

3 . Se dirigió el transductor hacia el hombro izquierdo, profundidad de $16-20 \mathrm{~cm}$ hasta localizar la silueta cardíaca.

4. Una vez localizada la vena cava se realizó la medición en modo $\mathrm{M}$ a $2 \mathrm{~cm}$ distal de su unión con el atrio derecho.

5. Medición perpendicular al eje largo justo proximal a la unión de la vena hepática que es aproximadamente 0,5 a $3 \mathrm{~cm}$ de la aurícula derecha.

6. Se realizaron medidas del diámetro máximo en inspiración (valor normal: 0-14 $\mathrm{mm}$ ) y espiración (valor normal: 15-20 mm). La diferencia entre los diámetros en espiración e inspiración se consideró colapsabilidad y el índice de colapsabilidad se definió como ((VCI e-VCI i) / VCI e) $\mathrm{x} 100^{5}$.

Los datos se recogieron en una ficha por paciente en la cual se registraron los siguientes datos: grupo, edad, FC, presión arterial sistólica, tipo de trauma (cerrado/penetrante), diámetro de vena cava en

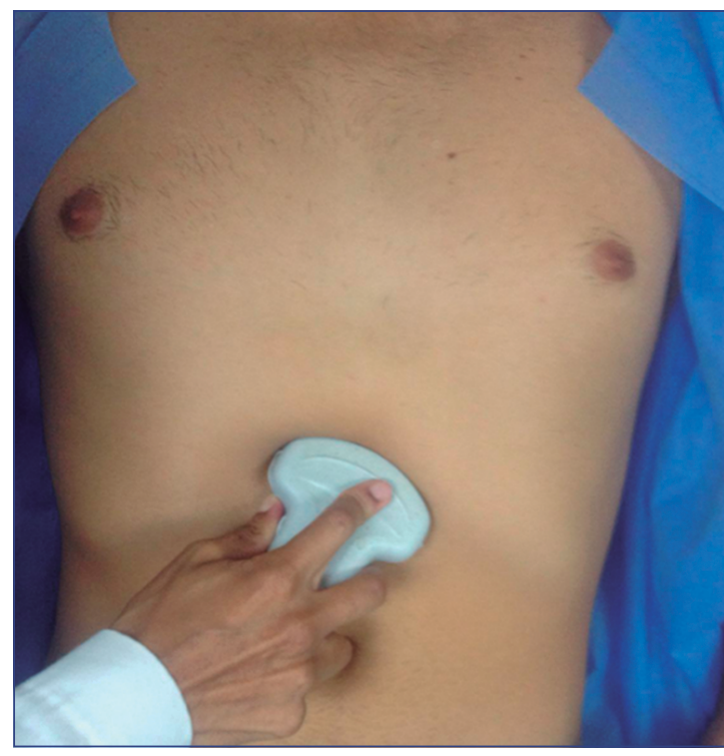

Figura 1. Localización de transductor convexo DE 2-5 MhZ en paciente para la medición ecográfica de la vena cava inferior. inspiración (VC i), diámetro de vena cava en espiración (VCI e), índice de colapsabilidad ((VCI e-VCI i)/VCI e) x 100. Según los valores de PAS, se determinó si el paciente presenta shock hipovolémico o no, utilizando la $\mathrm{PAS}<90 \mathrm{mmHg}$ como punto de corte, acumulando un total de 20 pacientes control y 20 pacientes chocados.

\section{Tratamiento estadístico}

El análisis estadístico propuesto calculó el promedio y la desviación estándar de las variables continuas y en el caso de las variables nominales, se calculó sus frecuencias y porcentajes. Los contrastes de las variables continuas entre los grupos se evaluaron con la prueba t de Student para muestras independientes, mientras que para las variables nominales se evaluaron con la prueba chi-cuadrado de Pearson. Se calculó la sensibilidad y especificidad de la medición ecográfica de la vena cava inferior para determinar el estado de shock en el paciente, siendo comparado con la medición de la PAS. Se consideró un valor estadísticamente significativo si $\mathrm{p}<0,05$. Los datos fueron analizados con el software SPSS Statistics 24.

\section{Resultados}

Cuatro pacientes menores de 16 años y tres pacientes que no eran adecuados para la visualización ecográfica de la VCI debido a dificultades técnicas fueron excluidos del estudio, y los 40 pacientes 
Figura 2. Vena cava inferior como factor predictor del shock en trauma: medición ecográfica. Diagrama de barra de la PAS según grupos.

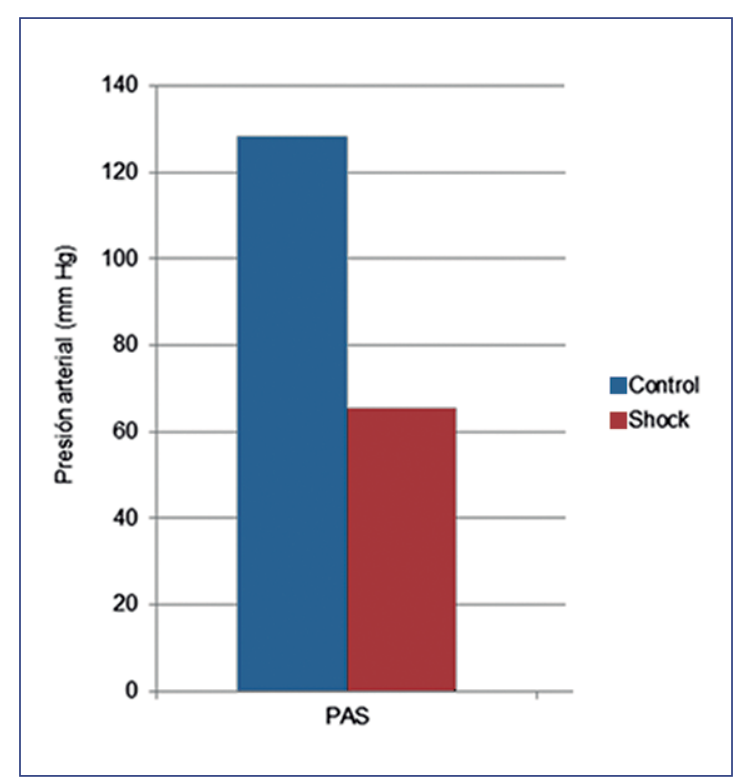

Tabla 1. Vena cava inferior como factor predictor del shock en trauma: medición ecográfica. Comparación de la media de los diámetros de la VCIe y VCIi en grupo control y de shock $(\mathrm{mm})$

\begin{tabular}{|lccc|}
\hline Grupos & n & VCIe & VCIi \\
\hline Shock* & 20 & $14,3 \pm 2,4$ & $6 \pm 1,6$ \\
\hline Control* $^{*}$ & 20 & $14,1 \pm 2$ & $10.4 \pm 2,1$ \\
Total* $^{*}$ & 40 & $14,2 \pm 2,2$ & $8,2 \pm 2,9$ \\
\hline
\end{tabular}

* Media \pm desviación estándar. $\mathrm{p}=0,001$.

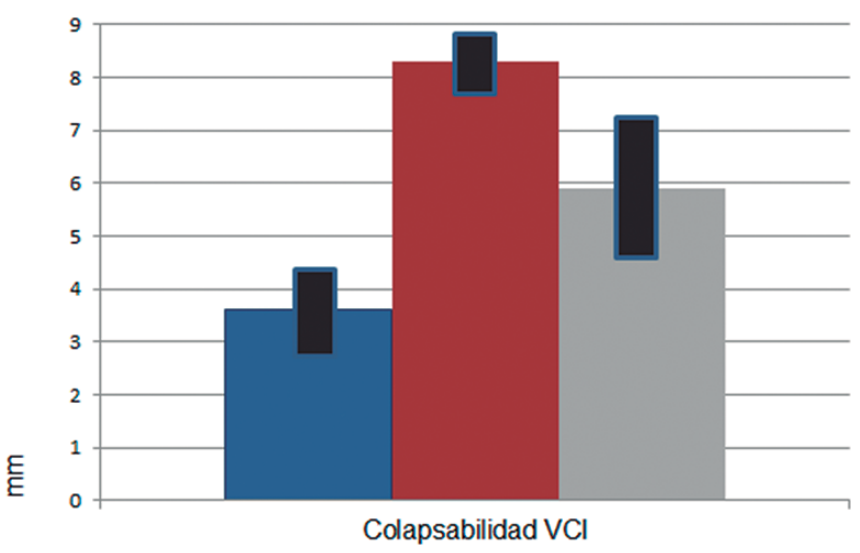

- Control - Shock - Total aE

DE = Desviación estándar

Figura 3. Vena cava inferior como factor predictor del shock en trauma: medición ecográfica. Colapsabilidad media de la VCl en grupo control y de shock. restantes fueron investigados. El rango de edad fue de 19 a 58 años en el grupo control y de 21 a 45 años en el grupo de shock. El único parámetro que fue diferente entre los dos grupos fue la presión arterial sistólica $(\mathrm{p}<0,001)$ (Figura 2). No hubo diferencias estadísticamente significativas en otros parámetros, incluidos la edad, el sexo y la presencia de un trauma penetrante. En el grupo de shock, todos los pacientes recibieron laparotomía de emergencia. Ningún paciente en el grupo de control recibió cirugía durante nuestro período de estudio. El tamaño de la VCI fue máximo con el paciente en posición supina y al final de la espiración. Con respecto a todos los pacientes, la media de los diámetros VCIe y VCIi fue de 14,2 $\pm 2,2 \mathrm{~mm}$ y $8,2 \pm 2,9 \mathrm{~mm}$, respectivamente (Tabla 1 ). La colapsabilidad media de la VCI en ambos grupos fue de $5,9 \pm 2,7 \mathrm{~mm}$, siendo para el grupo control de $3,6 \pm 1,7 \mathrm{~mm}$ y para el grupo de shock de $8,3 \pm 1,1$ mm (Figura 3). La media del Índice de colapsabilidad de la VCI para el grupo control y de shock fue de $26 \pm 12,7 \%$ y de $58,5 \pm 5,9 \%$, respectivamente (Tabla 2). Se determinó el punto de corte del Índice de colapsabilidad $>50 \%$ según ROC (Tabla 3 ). Los valores diagnósticos del índice de colapsabilidad de la VCI en relación a sensibilidad, especificidad, índice de validez, valor predictivo positivo, valor predictivo negativo y prevalencia se encuentran resumidos en la Tabla 4.

Tabla 2. Vena cava inferior como factor predictor del shock en trauma: medición ecográfica. Comparación del índice de colapsabilidad y presencia de shock

\begin{tabular}{|cccccc|}
\hline Grupos & n & Mínimo & Máximo & Media & DE \\
Shock & 20 & 47,4 & 71,4 & 58,5 & 5,9 \\
Control & 20 & 6,6 & 50,0 & 26,0 & 12,7 \\
\hline $\mathrm{p}=0,001$. & & & & & \\
\hline
\end{tabular}

Tabla 3. Vena cava inferior como factor predictor del shock en trauma: medición ecográfica. Relación del índice de colapsabilidad y presencia de shock

\begin{tabular}{|cccc|}
\hline $\begin{array}{c}\text { Índice de } \\
\text { colapsabilidad }\end{array}$ & \multicolumn{2}{c}{ Grupos } & Total \\
& Shock & Control & \\
$>50 \%$ & 20 & 0 & 20 \\
$\leq 50 \%$ & 0 & 20 & 20 \\
\hline Total & 20 & 20 & 40 \\
\hline
\end{tabular}

$\mathrm{p}=0,001$. Punto de corte del índice de colapsabilidad según ROC $>50 \%$. 
Tabla 4. Vena cava inferior como factor predictor del shock en trauma: medición ecográfica. Valores diagnósticos del índice de colapsabilidad en presencia de shock

\begin{tabular}{|lccc|}
\hline Indicadores & Valores & \multicolumn{2}{c|}{ IC - 95\% } \\
Sensibilidad & 100,0 & 95,0 & 100,0 \\
Especificidad & 100,0 & 97,5 & 100,0 \\
Índice de validez & 100,0 & 98,3 & 100,0 \\
VPP & 100,0 & 95,0 & 100,0 \\
VPN & 100,0 & 97,5 & 100,0 \\
Prevalencia & 33,3 & 14,8 & 51,9 \\
\hline
\end{tabular}

\section{Discusión}

El concepto de shock ha evolucionado lentamente a través de la historia ${ }^{6}$, hasta lo que conocemos hoy en día como: "La pérdida aguda de la adecuada capacidad de perfusión de los tejidos para alcanzar las necesidades metabólicas celulares". Considerando en un adulto la presión arterial sistólica (PAS) $<90$ $\mathrm{mmHg}$ o la presión arterial media $(\mathrm{PAM})<$ a 60 $\mathrm{mmHg}$ como valores de hipotensión severa los cuales pueden presentarse en el estado de shock ${ }^{7}$.

El shock hipovolémico, se caracteriza por un volumen de sangre circulante disminuidos, causando disminución de la precarga, por disminución del llenado ventricular, lo que produce reducción del volumen sistólico y gasto cardíaco ${ }^{6}$.

En el estudio realizado por Huang et al. ${ }^{8}$, la precisión diagnóstica del diámetro de la vena cava inferior en la predicción de la respuesta del fluido en pacientes con shock circulatorio que reciben ventilación mecánica fue moderadamente predictivo con una especificidad combinada de 0,80 (IC del 95\%, 0,66-0,89). En nuestro trabajo, todos los pacientes que ingresaron a la unidad de politraumatizados posterior a la medición de la presión arterial sistólica y medición del índice de la colapsabilidad de la VCI se les administro $2000 \mathrm{cc}$ de infusión endovenosa de líquidos y se evaluó la respuesta al mismo.

La VCI es considerada por Marc Feissel et al. , como un vaso de gran calibre, cuyo tamaño y dinámica varían con los cambios en el agua corporal total y las respiraciones. Aunque hay resultados contradictorios sobre las variaciones respiratorias en la apariencia ecográfica de la $\mathrm{VCI}^{10}$, la mayoría de los autores sienten que la VCI normal disminuirá en calibre con la inspiración y aumentará en calibre durante la espiración ${ }^{11}$. Nuestro estudio reveló que la
VCIi es significativamente más pequeña en pacientes con shock, un hallazgo que también se informó en estudios reciente ${ }^{12,13}$.

$\mathrm{Su}$ diámetro tiene una alta correlación con la función de las cavidades cardíacas derechas y su calibre no es afectado por la respuesta compensatoria vasoconstrictora que se genera ante la pérdida de volumen intravascular. Por eso, refleja el estatus de la volemia con mayor precisión que otros parámetros, como los basados en el sistema arterial (presión sanguínea, diámetro de la aorta, etc $)^{14}$.

Yanagawa et al. ${ }^{13}$, encontraron que el diámetro de la VCIe por debajo de $9 \mathrm{~mm}$ se asocia con la presencia de shock en pacientes traumatizados. Sin embargo en nuestro estudio el diámetro de la VCIe no obtuvo correlación como indicador de shock, en contraste con la VCIi y el IC $>50 \%$, siendo determinantes en ambos grupos. Por lo tanto, los hallazgos anteriores indican que IVCi puede ser un indicador más confiable que IVCe. Siendo el IC el mejor indicador pronóstico de shock en los pacientes estudiados.

Nuestros resultados son similares que los estudios de Sefidbakht et al. ${ }^{12}$ y Yanagawa et al. ${ }^{13}$, en relación a la presencia de un IC mayor en pacientes en shock. Sin embargo difieren en cuanto a los resultados de $\mathrm{VCIe}$, los cuales son significativamente menores en sus trabajos.

Este Trabajo tiene varias implicaciones clínicas. En primer lugar, muestra que la medición del diámetro de la VCI es un indicador sensible y especifico de shock en pacientes severamente lesionados e incluso puede predecirlo en pacientes que todavía tienen presión arterial normal debido a una hiperactividad simpática. Dos pacientes de la muestra que ingresaron a la unidad de Politraumatizados mostraron valores de presión de arterial normales al llegar, fueron monitoreados luego de la infusión de $2.000 \mathrm{cc}$ de solución fisiológica durante su estadía en las primeras $6 \mathrm{~h}$ en la unidad de politraumatizados, y en el transcurso presentaron deterioro de la cifras tensionales con un IC $>50 \%$ obteniendo una respuesta transitoria a la reanimación de líquidos ${ }^{1}$. En segundo lugar, las mediciones en serie del diámetro de la VCI se pueden usar para monitorear la pérdida de sangre en curso y monitorear la terapia de fluidos, e incluso se pueden usar como una alternativa para dirigir la medición de la presión venosa central, que no es adecuada como un procedimiento de rutina ${ }^{8,9}$. Ambos grupos en nuestro estudio mostraron índices de colapsabilidad de $\geq 50 \%$, y este índice fue mayor en el grupo de shock, lo que puede deberse a la disminución del volumen y la actividad cardíaca más intensa en este grupo. 


\section{Conclusiones}

La medición del índice de colapsabilidad de la VCI, es un factor predictor del shock en trauma, con una sensibilidad y especificidad de $100 \%$. Al usar la ecografía como método no invasivo, no existen riesgos ni complicaciones a diferencia de otros procedimientos invasivos. Pudiendo incluirse en el FAST al momento de la valoración primaria del paciente severamente lesionado, debido al poco tiempo necesario para realizarse, aumentando la supervivencia y disminuyendo las complicaciones.

\section{Responsabilidades éticas}

Protección de personas y animales. Los autores declaran que para esta investigación no se han realizado experimentos en seres humanos ni en animales, bajo los principios éticos para las investigaciones médicas en seres humanos según la declaración de Helsinki.

Confidencialidad de los datos. Los autores declaran que en este artículo no aparecen datos de pacientes.

\section{Conflicto de Intereses: Ninguno.}

\section{Referencias}

1. Ottolino P, Vivas R. Manejo integral del paciente politraumatizado. Caracas: Panamericana; 2008.

2. Orso D, Paol I, Piani T, Cilenti F, Cristiani L, Guglielmo N. Accuracy of Ultrasonographic Measurements of Inferior Vena Cava to Determine Fluid Responsiveness: A Systematic Review and Meta-Analysis. Department of Emergency Medicine, Udine, Italy. J Intensive Care Med. 2018 Jan 1:885066617752308. doi: $10.1177 / 0885066617752308$.

3. McGee DC, Gould MK. Preventing complications of central venous catheterization. N Engl J Med. 2003;348:1123-33.

4. Seif D, Mailhot T, Perera P. Caval sonography in shock a noninvasive method for evaluating intravascular volume in critically ill patients. $\mathrm{J}$ Ultrasound Med. 2012;31:1885-90.

5. Esper R, Velasco R, Talamantes Y
Evaluación de la precarga y respuesta a volumen mediante ultrasonografía de la vena cava. Rev Asoc Mex Med Crit y Ter Int. 2015;2:105-12.

6. Cantele H, Méndez M. Semiología y patología quirúrgica. Caracas: Mc Graw Hill; 2003.

7. Ceraso D. Hipotensión Arterial y Shock. Hospital Juan A. Fernández, Buenos Aires, Argentina. Federación Argentina de Cardiología, 2001.

8. Huang H, Qinkang S, Yafen L, Hua X, Yaxin F. Value of variation index of inferior vena cava diameter in predicting fluid responsiveness in patients with circulatory shock receiving mechanical ventilation: a systematic review and meta-analysis. Critical Care 2018 22:204. Published online 2018 Aug 21. doi: 10.1186/s13054-018-2063-4.

9. Marc F, Michard F, Faller JP. The respiratory variation in inferior vena cava diameter as a guide to fluid therapy. Int Care Med. 2014;30:1834-7.
10. Akilli B, Bayir A, Kara F. Inferior vena cava diameter as a marker of early hemorrhagic shock: a comparative study. Ulus Trauma Acil Cerrahi Der. 2010;2:113-8.

11. Dipti A, Soucy Z, Surana A. Role of inferior vena cava diameter in assessment of volumen status: a meta-analysis. Am J Emerg Med. 2012;30:1414-9.

12. Sefidbakht $S$, Assadsangabi R, Abbasi HR. Sonographic measurement of the inferior vena cava as a predictor of shock in trauma patients. Emerg Radiol. 2007;14:181-5.

13. Yanagawa Y, Sakamoto T, Okada Y. Hypovolemic shock evaluated by sonographic measurement of the inferior vena cava during resuscitation in trauma patients. J Traum. 2007;63:1245-8.

14. Iturbide I, Santiago ML, Henain F. Evaluación ecográfica de la vena cava inferior en los pacientes hemodinámicamente inestables. Rev Argent Radiol. 2017;3:209-13. 